Max-Planck-Institut für demografische Forschung

Max Planck Institute for Demographic Research

Konrad-Zuse-Strasse 1 - D-18057 Rostock - GERMANY

Tel +49 (0) 3812081 - 0; Fax +49 (0) 3812081 - 202;

http://www.demogr.mpg.de

MPIDR WORKING PAPER WP 2010-002

JANUARY 2010 (REVISED FEBRUARY 2010)

\title{
Zur Sterblichkeitsdifferenz von \\ Männern im Ost-West-Vergleich
}

\author{
Rembrandt Scholz (scholz@demogr.mpg.de) \\ Anne Schulz \\ Michael Stegmann
}

This working paper has been approved for release by: Mikołaj Szołtysek (szoltysek@demogr.mpg.de),

Deputy Head of the Laboratory of Historical Demography.

(C) Copyright is held by the authors.

Working papers of the Max Planck Institute for Demographic Research receive only limited review. Views or opinions expressed in working papers are attributable to the authors and do not necessarily reflect those of the Institute. 


\title{
Zur Sterblichkeitsdifferenz von Männern im Ost-West-Vergleich
}

\author{
Rembrandt Scholz* / Anne Schulz** Michael Stegmann*** \\ *Max-Planck-Institut für demografische Forschung, Rostock \\ ${ }^{*}$ Rostocker Zentrum zur Erforschung des Demografischen Wandels \\ ${ }^{* \star *}$ Deutsche Rentenversicherung Bund, Forschungsdatenzentrum der Rentenversicherung (FDZ-RV), \\ Würzburg
}

Abstract: Die Untersuchung der Sterblichkeit der sozialversicherungspflichtigen Beschäftigten des Jahres 2004 deckt die Gründe für die Unterschiede der Lebenserwartung bei Männern im Ost-West-Vergleich auf. Es wird gezeigt, dass die bestehenden strukturellen Unterschiede des Arbeitsmarktes in Ost- und Westdeutschland die Lebenserwartungsdifferenz bei Männern im Alter bis 65 Jahre erklären können.

Die multivariate Analyse von Individualdaten der 20 Millionen „Aktiv Versicherten“ (Datensätze des Forschungsdatenzentrum der Rentenversicherung, FDZ-RV) zeigt, dass die Berücksichtigung der Merkmale Beschäftigung, Arbeitslosigkeit, Selbstständigkeit, Versicherungsart und Staatsbürgerschaft geeignet ist, eine bis zu $30 \%$ erhöhte Sterblichkeit der ostdeutschen Männer im Altersbereich von 35 bis 55 Jahre zu erklären. Die Differenzen sind wahrscheinlich ein Ergebnis der unterschiedlichen Arbeitsmarktlage und der damit verbundenen unterschiedlichen Komposition der beiden Teilbevölkerungen.

\section{Schlüsselworte:}

Arbeitslosigkeit, Lebenserwartung, logistisches Regressionsmodell, Männer, Ost-West- Differenz 


\section{Einleitung}

Im Zeitraum der letzten 50 Jahre kommt es in Deutschland zu einer sehr starken Zunahme der Lebenserwartung. Besonders bemerkenswert ist der Anstieg der Lebenserwartung in Ostdeutschland seit 1990. Innerhalb von 15 Jahren ist eine Zunahme von 6 bis 7 Lebensjahren zu verzeichnen. Die Angleichung der Lebensbedingungen und insbesondere der medizinischen Versorgung in Ost- und Westdeutschland führen zur Angleichung der Lebenserwartung (Wiesner und Bittner 2004; Kibele und Scholz 2009 a, b). Vor der deutschen Einheit betrug die höchste Ost-West-Differenz der Lebenserwartung etwa 3 bis 4 Lebensjahre. Diese betraf alle Altersgruppen, aber besonders das höhere Alter ab 65 Jahre. Nach 1990 kommt es im höheren Alter sehr schnell zu einer Annäherung der Lebenserwartung, was sich auf alle Altersklassen ab dem Alter 60 bezieht und für beide Geschlechter gilt.

Bei Frauen ist die Lebenserwartung heute insgesamt bei etwa 82,5 Lebensjahren angelangt und befindet sich in Ost- und Westdeutschland auf dem gleichen Niveau. Bei den Männern besteht eine Differenz von 1,5 Lebensjahren. Diese Differenz wird vor allem von den Altersklassen der 35- bis 55-jährigen Männer getragen, Männer in Westdeutschland werden durchschnittlich 78 Jahre alt und die Lebenserwartung der ostdeutschen Männer beträgt 76,5 Jahre (Human Mortality Database, www.mortality.org).

Viele Studien haben bisher zeigen können, dass Mortalitätsunterschiede zwischen verschiedenen Gruppen oftmals durch sozioökonomische Differenzen erklärt werden können. Vor allem für Männer kann der Einfluss sozioökonomischer Determinanten auf die Sterblichkeit nachgewiesen werden. So stellt Klein (1993) für Männer einen deutlichen Gradienten der Lebenserwartung hinsichtlich der Arbeitsbedingungen heraus. Männer in einer höheren beruflichen Stellung weisen ein weitaus geringeres Sterberisiko auf als Männer in einer niedrigen beruflichen Stellung. Luy (2006) kann mit Daten des Lebenserwartungssurveys zeigen, dass für Männer im Alter von 45 Jahren deutliche Unterschiede in der Restlebenserwartung bestehen, wenn man nach Berufsgruppen, Bildung und Nettoeinkommen differenziert. So haben Männer in der höchsten Berufs-, Bildungs- bzw. Einkommensgruppe eine höhere Restlebenserwartung im Vergleich zu ihren Altersgenossen. Klein und Unger (2001) heben den engen Zusammenhang von Bildung, Einkommen und beruflicher Stellung und deren kumulierten Einfluss auf die Sterblichkeit hervor. Sie argumentieren, dass die Schichtabhängigkeit des Einkommens und die damit verbundenen ungesünderen Arbeitsbedingungen in den unteren sozialen Schichten zu den Differenzen in der Lebenserwartung 
führen. Geringere Bildung ist verbunden mit geringerem Einkommen und ungesünderem Verhalten wie höherer Raucherprävalenz; andere Einflüsse sind mit den Ernährungs- und Wohnbedingungen verbunden. Außerdem ist der Einkommenseffekt auch beim Zugang zum medizinischen Versorgungssystem und der Inanspruchnahme medizinischer Leistungen zu vermuten.

Die Auswahl der Studien zeigt, wie wichtig sozioökonomische Faktoren sind, wenn man den Sterblichkeitsunterschied zwischen verschiedenen sozioökonomischen Gruppen untersuchen will. So wird vermutet, dass sich auch die Mortalitätsdifferenzen zwischen Ost- und Westdeutschland durch eine unterschiedliche sozioökonomische Struktur der beiden Regionen erklären lassen. Für diesen Ansatz hat Grözinger (2009) einen Literaturüberblick gegeben und gleichzeitig mit ökologischen Analysen der Raumordnungsregionen den Zusammenhang von Arbeitslosigkeit und Lebenserwartung untersucht. Analysen zum Zusammenhang von Arbeitslosigkeit und Lebenserwartung anhand von Daten der Rentenversicherung belegen sowohl Kibele (2008) als auch Scholz und Schulz (2009).

Die Daten vom Forschungsdatenzentrum der Rentenversicherung (FDZ-RV) haben sich als valide erwiesen, die Mortalität der Männer ab dem Alter 65 in Deutschland abzubilden (Shkolnikov et al. 2008; Himmelreicher et al. 2008; Scholz und Schulz 2008), da fast alle Männer in Deutschland Leistungen von der gesetzlichen Rentenversicherung beziehen. Für Personen im Alter unter 65 Jahre bezieht nur ein sehr kleiner Teil der Bevölkerung eine Rente und dieser Teil ist stark gesundheitlich beeinträchtigt, wodurch Aussagen zur Mortalität der Bevölkerung eingeschränkt sind. Die Deutsche Rentenversicherung verfügt durch die Abführung von Rentenversicherungsbeiträgen im Datensatz „Aktiv Versicherte“ über monatliche Informationen von allen abhängig Beschäftigten. Es kann von den Personen festgestellt werden, ob sie sich im Anfangs- und Endbestand eines Jahres der „Aktiv Versicherten“ befinden oder ob ein Sterbefall aufgetreten ist. Diese Daten sind für eine Mortalitätsanalyse geeignet, da die Auswertung auf einer Personenstatistik aufbaut, bei der keine Verzerrungen des Bestandes auftreten können. Die Statistik beruht auf Geldzahlungen und ist mit einer eineindeutigen Zuordnung verbunden. Es können Merkmale des Versichertenkontos als anonymisierter Datensatz des Forschungsdatenzentrums der Rentenversicherung (FDZ-RV) in die Analyse einbezogen werden. Die Daten umfassen alle Personen in abhängiger Beschäftigung, die Rentenbeiträge zahlen und stellen die Grundlage der Mortalitätsanalyse dar. 


\section{Material und Methode}

Für die Analyse werden die Daten der „Aktiv Versicherten“ des Jahres 2004 der Deutschen Rentenversicherung herangezogen. Die Statistik der „Aktiv Versicherten“ wird auf Grundlage des $\S 79$ des Vierten Buches Sozialgesetzbuch (SGB IV) erhoben und ist eine Fortentwicklung der früheren Pflichtversichertenstatistik. Ihre Durchführung ist in $\S 1$ Abs. 1 der „Allgemeinen Verwaltungsvorschrift über die Statistik in der Rentenversicherung (RSVwV)“ vom 30. Januar 1992 geregelt. Der Versichertenbestand ist für die gesetzliche Rentenversicherung eine der entscheidenden Größen, von der die Beitragseinnahmen und die zukünftigen Belastungen durch Rehabilitationsleistungen und Renten abhängen. Die Statistik der „Aktiv Versicherten“ nach § 1 Abs. 1 RSVwV informiert insbesondere die Rentenversicherungsträger, Aufsichtsbehörden, Bundesministerien, Sozialministerien der Länder, die Deutsche Rentenversicherung und andere Beteiligte bzw. Interessierte über die Anzahl und Struktur der in der gesetzlichen Rentenversicherung versicherten Personen. Der Berichtszeitraum umfasst das durch den Erhebungsstichtag abgeschlossene Jahr als Berichtsjahr, sowie das davor liegende Jahr mit dem 31.12. des Berichtsvorjahres.

Für die Analysen zur Sterblichkeit wurden die Mikrodaten des aus dem Bestand der „Aktiv Versicherten“ des Jahres 2004 vom FDZ-RV in aggregierter Form als Sonderdatenauswertung zur Verfügung gestellt. Bei der Zusammenstellung der Daten und der darauf basierenden Analyse muss man folgende Restriktionen ins Kalkül ziehen:

Da es sich um eine Statistik der „Aktiv Versicherten“ handelt, sind Personen ausgeschlossen, die keine rentenrechtlichen Zeiten aufweisen, weil sie bereits vorher Rentenbezieher waren (insbesondere Erwerbsminderungsrentner oder ab dem Alter 60 auch Altersrentner; aber auch alle, die aus sonstigen Gründen keine versicherungsrelevanten Zeiten haben). Das heißt, dass gesundheitlich schlechter Gestellte eines Jahrganges systematisch ausgeschlossen werden. Ebenso nicht erfasst sind Personen ohne Kontakt zur Rentenversicherung, darunter z. B. so genannte gute Risiken wie berufständisch gesicherte Personen (beispielsweise Ärzte, Rechtsanwälte, Architekten). Diese Restriktionen in Kauf nehmend, kann man folgenden Ansatz wählen:

a) Bestimme als Ausgangspopulation die Personen, die im Jahr t-1 eine rentenrechtliche Zeit aufweisen und

b) identifiziere als Abgangspopulation die Personen, auf die a) zutrifft und die im Berichtsjahr t verstorben sind. 
Dieser Vorgabe folgend wurden für die Aggregationsmerkmale Alter, Beschäftigung, Versicherung und Staatsangehörigkeit die Bestands- und Abgangsmengen bestimmt und als Sonderauswertung zur Verfügung gestellt. Die Analyse bezieht sich auf alle 20 Millionen „Aktiv Versicherte“ Männer in Deutschland im Alter bis 64 Jahre und deren 154.000 Sterbefälle.

Aus den Angaben zu den Versicherungsbeständen nach Alter und Region am 1.1.2004 und den Sterbefällen des Jahres 2004, der Personen die am 1.1.2004 zum Bestand der Aktiv Versicherten gehört haben, werden altersspezifische Sterbewahrscheinlichkeiten ermittelt. Anschließend werden die ostdeutschen altersspezifischen Sterbewahrscheinlichkeiten durch die westdeutschen altersspezifischen Sterbewahrscheinlichkeiten geteilt:

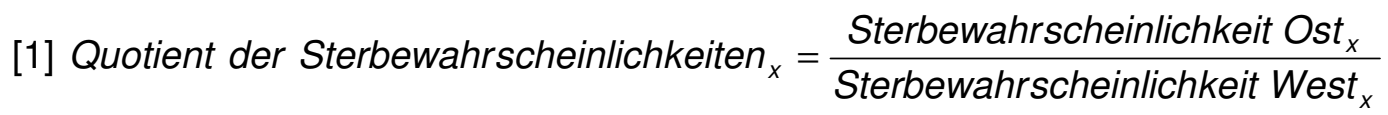

Nimmt der Quotient einen Wert über 1 an, spricht dies für eine Übersterblichkeit in Ostdeutschland. Liegt der Wert unterhalb von 1, besteht eine höhere Sterblichkeit in Westdeutschland. Diese Berechnung wird in den folgenden Ausführungen für alle Untergruppen nach Zeiten der Beschäftigung und der Art der Versicherung vorgenommen.

Nach der bivariaten Analyse der ostdeutschen Übersterblichkeit wird versucht zu erklären, welche Faktoren den Ost-West-Unterschied in der Sterblichkeit bedingen. Dazu wird ein logistisches Regressionsmodell (Hosmer und Lemeshow 1989) gerechnet, welches den Überlebensstatus der Aktiv Versicherten des Jahres 2004 zur abhängigen, dichotomen Variable hat. Es wird das relative Sterberisiko in Abhängigkeit von der Region $R$, vom Alter $A$, vom Beschäftigungsstatus $B$, vom Versicherungsstatus $V$ sowie von der Staatsangehörigkeit $S$ geschätzt:

[2] $\ln \left[\frac{\pi(x)}{1-\pi(x)}\right]=C+\sum_{i=1}^{2} \delta_{i} \cdot R_{i}+\sum_{j=20-24}^{60-64} \gamma_{j} \cdot A_{j}+\sum_{k=1}^{3} v_{k} \cdot B_{k}+\sum_{l=1}^{2} \lambda_{l} \cdot V_{l}+\beta \cdot S$

Anschließend wird das vorliegende Modell um einen Interaktionseffekt ergänzt, der die Interaktion zwischen der Region und den Altersgruppen abbildet. 


\section{Ergebnisse}

Der folgende Abschnitt beschreibt die Ergebnisse der Analysen zur ostdeutschen Übersterblichkeit. Tabelle 1 gibt an, wie sich das Verhältnis der altersspezifischen Sterbewahrscheinlichkeiten von Ost- zu Westdeutschland insgesamt, nach den Zeiten der Beschäftigung und nach der Art der Versicherung darstellt. Diese beiden Merkmale dienen hier als Indikatoren für sozioökonomische Determinanten wie Schichtzugehörigkeit, berufliche Stellung, Bildung und Einkommen. Unabhängigkeit von diesen Merkmalen zeigt sich, dass bis auf die Altersgruppe der 60- bis 64-Jährigen in allen Altersgruppen eine Übersterblichkeit der Ostdeutschen vorliegt, wobei diese zwischen Alter 30 und 54 besonders stark ausgeprägt ist. Hier weisen die ostdeutschen Männer eine um $20 \%$ bis $54 \%$ höhere Sterbewahrscheinlichkeit auf als ihre westdeutschen Altersgenossen. Um zu verifizieren, welche Untergruppen dieses Phänomen tragen, wird nach Zeiten der Beschäftigung und nach Art der Versicherung differenziert. So wird zwischen Personen unterschieden, die in der Vorjahresperiode nur Zeiten der abhängigen Beschäftigung hatten (60\%) und Personen, die neben der abhängigen Beschäftigung auch oder nur Zeiten der Arbeitslosigkeit (20,5\%) oder der Selbstständigkeit $(0,5 \%)$ aufwiesen. Weiterhin gibt es eine Gruppe von Personen, für die keine Angaben zu ihren Beschäftigungszeiten vorliegen, da sie aus anderen Gründen rentenversicherungspflichtig waren (19\%).

Es zeigt sich, dass die ostdeutsche Übersterblichkeit in der Gruppe der Personen, die nur Zeiten in abhängiger Beschäftigung aufweisen, am geringsten ausfällt. Für Personen, die Zeiten in abhängiger Beschäftigung und bzw. nur Zeiten in Selbstständigkeit aufweisen, ist kein kontinuierlicher Verlauf über die Altersgruppen hinweg zu beobachten. Altersgruppen mit hoher ostdeutscher Übersterblichkeit werden von Altersgruppen mit hoher westdeutscher Übersterblichkeit gefolgt. Diese Schwankungen sind sicherlich auf die geringe Fallzahl zurückzuführen. Besonders hohe Werte der Übersterblichkeit sind in der Gruppe zu beobachten, für die keine Angabe zu diesem Merkmal vorhanden ist. Hier übersteigen die ostdeutschen Sterbewahrscheinlichkeiten zum Teil bis zu $90 \%$ die Sterbewahrscheinlichkeiten aus Westdeutschland. In dieser Gruppe lassen sich Personen mit einer geringfügigen Beschäftigung vermuten. Die Differenzierung nach der Versicherung kann als Proxy für die Art der Tätigkeit angesehen werden. Es wird zwischen der Arbeiterrentenversicherung, der Angestelltenversicherung und der Knappschaft unterschieden. Personen, die der Arbeiterrentenversicherung angehören, bilden mit knapp 60 \% die größte Gruppe. Der Angestellten- 
versicherung gehören in etwa $37 \%$ an und die Knappschaft stellt mit fast $3 \%$ die kleinste Gruppe dar.

Tabelle 1: Quotienten der altersspezifischen Sterbewahrscheinlichkeiten Ost - zu Westdeutschland nach Gruppen der Beschäftigung und Versicherung, „Aktiv Versicherte" Männer 2004

\begin{tabular}{|c|c|c|c|c|c|c|c|c|c|c|c|}
\hline \multicolumn{2}{|r|}{ Alter } & bis 19 & $20-24$ & $25-29$ & $30-34$ & $35-39$ & $40-44$ & $45-49$ & $50-54$ & $55-59$ & $60-64$ \\
\hline \multicolumn{2}{|c|}{$\begin{array}{c}\text { Quotient der } \\
\text { Sterbewahrscheinlichkeiten } \\
\text { Ost zu West }\end{array}$} & 1.528 & 1.087 & 1.089 & 1.205 & 1.494 & 1.541 & 1.486 & 1.274 & 1.128 & 0.933 \\
\hline \multirow[t]{3}{*}{$\begin{array}{c}\text { Zeiten } \\
\text { der } \\
\text { Beschäfti- } \\
\text { gung }\end{array}$} & $\begin{array}{c}\text { nur } \\
\text { Beschäftigungs- } \\
\text { zeiten }\end{array}$ & 0.885 & 1.103 & 1.070 & 1.186 & 1.065 & 1.090 & 0.989 & 0.980 & 0.890 & 0.847 \\
\hline & $\begin{array}{c}\text { Zeiten der } \\
\text { Beschäftigung und } \\
\text { Arbeitslosigkeit }\end{array}$ & 0.739 & 0.956 & 0.895 & 0.906 & 1.307 & 1.326 & 1.330 & 1.065 & 1.025 & 0.967 \\
\hline & $\begin{array}{c}\text { Zeiten der } \\
\text { Beschäftigung und } \\
\text { Selbstständigkeit }\end{array}$ & - & - & - & 0.501 & 1.082 & 1.601 & 0.749 & 1.787 & 1.581 & 1.119 \\
\hline
\end{tabular}

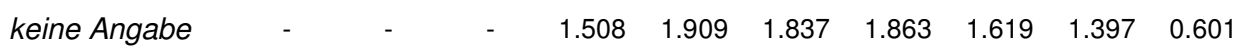

\begin{tabular}{|c|c|c|c|c|c|c|c|c|c|c|c|}
\hline $\begin{array}{l}\text { Art der } \\
\text { Versiche- } \\
\text { rung }\end{array}$ & $\begin{array}{c}\text { Arbeiterrenten- } \\
\text { versicherung }\end{array}$ & 1.501 & 1.033 & 0.997 & 1.104 & 1.401 & 1.433 & 1.448 & 1.252 & 1.149 & 0.928 \\
\hline & $\begin{array}{l}\text { Angestellten- } \\
\text { versicherung }\end{array}$ & 0.695 & 1.071 & 1.136 & 1.181 & 1.131 & 1.266 & 1.017 & 1.049 & 0.925 & 0.908 \\
\hline & Knappschaft & - & 0.395 & 2.390 & 0.874 & 1.401 & 1.334 & 1.280 & 0.990 & 1.036 & 0.822 \\
\hline
\end{tabular}

Quelle: FDZ-RV - Aktiv Versicherte 2004; „Ausgang_AKVS2004n.dat“ und „Abgang_AKVS2004n.dat“, Sonderauswertung, eigene Berechnungen.

Innerhalb dieser Untergruppen ist festzustellen, dass für Personen in der Angestelltenversicherung die ostdeutsche Übersterblichkeit die geringsten Werte annimmt. Für die 20- bis 54-Jährigen haben die Ostdeutschen eine $5 \%$ bis $27 \%$ höhere Sterblichkeit als die Westdeutschen. In der Gruppe der Arbeiterrentenversicherung zeigt sich die ostdeutsche Übersterblichkeit sehr viel ausgeprägter. Hier fallen die ostdeutschen Sterbewahrscheinlichkeiten bis zu 50 \% höher aus als die westdeutschen altersspezifischen Sterbewahrscheinlichkeiten. Die kleinste Gruppe, die in der Knappschaft versicherten Beschäftigten, ist durch große Schwankungen zwischen den Altersgruppen gekennzeichnet. Besonders die Altersgruppe der 25- bis 29-Jährigen ist hier hervor- 
zuheben. In dieser Altersgruppe liegt in Ostdeutschland eine 2,4-fach höhere Sterbewahrscheinlichkeit im Vergleich zu Westdeutschland vor. Aber auch hier sei noch mal auf die geringe Fallzahl dieser Gruppe hingewiesen.

Insgesamt lässt sich nach dieser deskriptiven Analyse festhalten, dass die ostdeutsche Übersterblichkeit nicht in allen Altersgruppen und nicht in allen beschriebenen Untergruppen gleichermaßen auftritt. Besonders die mittleren Altersgruppen sind durch eine deutlich höhere Sterbewahrscheinlichkeit in Ostdeutschland im Vergleich zu Westdeutschland gekennzeichnet. Für die Untergruppen nach den Zeiten der Beschäftigung zeigt sich, dass vor allem die Gruppen, die Zeiten der Arbeitslosigkeit oder Selbstständigkeit aufweisen, sowie die Personen, für die keine Angabe zu diesem Merkmal vorliegt, die Übersterblichkeit in Ostdeutschland tragen. Die Differenzierung nach dem Versicherungszweig kann zeigen, dass die ostdeutsche Übersterblichkeit vornehmlich von den Angehörigen der Arbeiterrentenversicherung und der Knappschaft getragen wird.

Tabelle 2: Logistische Regressionsmodelle, relative Risiken der Sterblichkeit nach Region, Alter, Beschäftigung, Versicherung und Staatsbürgerschaft, „Aktiv Versicherte" Männer 2004

\begin{tabular}{|c|c|c|c|c|}
\hline Variable & Modell 1 & Modell 2 & Modell 3 & Modell 4 \\
\hline \multicolumn{5}{|l|}{ Region } \\
\hline West & 1,000 & 1,000 & 1,000 & 1,000 \\
\hline Ost & $1,357^{* * *}$ & $1,258^{* * *}$ & $1,070 * * *$ & $1,021^{* *}$ \\
\hline keine Angabe & 104,140 *** & $65,344^{* \star *}$ & $84,145^{* *}$ & $85,123^{* * *}$ \\
\hline \multicolumn{5}{|l|}{ Altersgruppe } \\
\hline bis 19 & & 1,000 & 1,000 & 1,000 \\
\hline $20-24$ & & $1,130 * \star *$ & 0,938 * & 0,948 \\
\hline $25-29$ & & $1,117^{\star * *}$ & 0,960 & 0,997 \\
\hline $30-34$ & & 1,408 *** & 1,248 *** & $1,299 * * *$ \\
\hline $35-39$ & & $2,043^{* \star *}$ & $1,841^{\star * *}$ & $1,889^{* * *}$ \\
\hline $40-44$ & & 3,396 *** & $3,036^{* * *}$ & $3,091^{* * *}$ \\
\hline $45-49$ & & $5,656^{* * *}$ & $5,085 * * *$ & $5,156 * * *$ \\
\hline $50-54$ & & $8,382^{\star * *}$ & $7,573^{* \star *}$ & $7,678^{* * *}$ \\
\hline $55-59$ & & $10,446^{\star \star \star}$ & $9,423^{\star \star *}$ & 9,682 *** \\
\hline $60-64$ & & $10,447^{* \star *}$ & $10,282 * * *$ & $10458 * * *$ \\
\hline \multicolumn{5}{|l|}{ Beschäftigung } \\
\hline nur Beschäftigungszeiten & & & 1,000 & 1,000 \\
\hline Beschäftigung und Arbeitslosigkeit & & & $2,014^{* * *}$ & $2,041^{* * *}$ \\
\hline Beschäftigung und Selbstständigkeit & & & 0,958 & 0,936 \\
\hline keine Angabe & & & $0,861^{* * *}$ & $0,860 * * *$ \\
\hline \multicolumn{5}{|l|}{ Versicherung } \\
\hline Arbeiterrentenversicherung & & & 1,000 & 1,000 \\
\hline Angestelltenversicherung & & & $0,567^{* * *}$ & $0,545 * * *$ \\
\hline Knappschaft & & & $0,924^{* * *}$ & $0,903^{* * *}$ \\
\hline \multicolumn{5}{|l|}{ Staatsangehörigkeit } \\
\hline deutsch & & & & 1,000 \\
\hline nicht deutsch & & & & $0,625 * * *$ \\
\hline Teststatistik G & $1.229 .853^{\star * \star}$ & $61.318^{* \star *}$ & $19.433^{\star \star *}$ & $1.653^{* * *}$ \\
\hline$d f$ & 2 & 9 & 5 & 1 \\
\hline
\end{tabular}

Quelle: FDZ-RV - Aktiv Versicherte 2004; „Ausgang_AKVS2004n.dat“ und „Agang_AKVS2004n.dat“, Sonderauswertung, eigene Berechnungen. 
Da die soeben vorgestellte deskriptive Analyse keine Aussage erbringen kann, inwiefern die gleichzeitige Wirkung der oben genannten Merkmale das Phänomen der ostdeutschen Übersterblichkeit beeinflusst, werden logistische Regressionsmodelle gerechnet.

Die erklärenden Variablen werden schrittweise in das Modell eingeführt, um den Effekt jeder einzelnen Variablen auf den Sterblichkeitsunterschied zwischen Ost- und Westdeutschland beurteilen zu können. Es gibt auch eine Gruppe von Personen, für die keine Angabe vorliegt, ob sie in Ost- oder Westdeutschland leben. Diese Gruppe wird, damit keine Verzerrungen auftreten, in die Analysen mit eingeschlossen. Auf eine Interpretation der Koeffizienten soll hier aber verzichtet werden. Die Teststatistik G, ein Maß zur Beurteilung eines logistischen Regressionsmodells, gibt an, ob die Einführung jeder erklärenden Variable das Modell signifikant verbessern kann. Ist dieser Wert signifikant, erhöht die Berücksichtigung einer neuen Variablen den Erklärungswert des Modells.

Tabelle 2 beinhaltet die Ergebnisse der Schätzungen. Modell 1 berücksichtigt nur die Region als erklärende Variable. Es ist zu sehen, dass Ostdeutsche im Vergleich zu Westdeutschen ein um 35,7\% höheres Sterberisiko aufweisen. Kontrolliert man für die Altersgruppen (Modell 2) zeigt sich, dass sich der relative Sterblichkeitsunterschied zwischen Ost- und Westdeutschland reduziert. Das Sterberisiko steigt, wie erwartet, mit dem Alter an. Es ist daher davon auszugehen, dass die ostdeutsche Bevölkerung eine ältere Altersstruktur als die westdeutsche Bevölkerung besitzt. Hier handelt es sich um einen Kompositionseffekt, der einen Teil der ostdeutschen Übersterblichkeit erklären kann.

In Modell 3 werden zusätzlich zum Alter der Beschäftigungsstatus und der Versicherungsstatus berücksichtigt. Hinsichtlich des Beschäftigungsstatus zeigt sich, dass Personen, die Beschäftigungszeiten hatten und arbeitslos waren, ein 2-fach höheres Sterberisiko haben als Personen, die ausschließlich Beschäftigungszeiten hatten. Arbeitslosigkeit scheint sich somit negativ auf die Sterblichkeit auszuwirken. Wurden neben Zeiten der abhängigen Beschäftigung auch Zeiten der Selbstständigkeit registriert, ergibt sich kein Sterblichkeitsunterschied zu Personen in der Referenzgruppe. Personen ohne Angabe zu ihrem Beschäftigungsstatus weisen ein um 13,9\% geringeres Sterberisiko auf als Personen, die nur Beschäftigungszeiten im Jahr 2004 hatten. Im Hinblick auf den Einfluss der Zugehörigkeit zu einer Versicherung zeigt sich, dass im Vergleich zu Personen, die der Arbeiterrentenversicherung angehören, Versicherte in der Angestelltenversicherung bzw. in der Knappschaft einem um 43,3\% bzw. 7,6\% reduzierten Sterberisiko ausgesetzt sind. 
Die Berücksichtigung der beiden Merkmale Beschäftigung und Versicherung führt dazu, dass sich wiederum der relative Sterblichkeitsunterschied zwischen Ost- und Westdeutschland verringert. In Ostdeutschland ist nun ein um $7 \%$ höheres Sterberisiko zu beobachten. Somit scheint Ostdeutschland auch hinsichtlich der Merkmale Beschäftigung und Versicherung (als Proxy für die Art der Tätigkeit) einer ungünstigeren Zusammensetzung ausgesetzt zu sein.

Modell 4, welches zusätzlich die Staatsangehörigkeit aufnimmt, zeigt, dass Personen, die keine deutsche Staatbürgerschaft besitzen, ein um 37,5\% geringeres Sterberisiko aufweisen als Personen mit deutscher Staatsbürgerschaft. Wird die Staatsanghörigkeit in das Modell eingeführt, verringert sich der relative Sterblichkeitsunterschied zwischen Ost- und Westdeutschland auf $2 \%$. Dies ist darauf zurückzuführen, dass der Ausländerteil in Ostdeutschland geringer ist und der positive Effekt auf die gesamte Sterblichkeit in Ostdeutschland geringer ist als in Westdeutschland. Das Ergebnis steht im Gegensatz zu der Studie von Shkolnikov et al. (2008). Hier führte die Einführung weiterer sozioökonomischer Variablen nicht zu einer Verringerung der Ost-WestSterberisiko-Differenz.

Die Auswertung des Interaktioneffektes, welcher gleichzeitig den Einfluss der Variablen „Beschäftigung“, „Versicherung“ und „Staatsangehörigkeit“ berücksichtigt, zeigt, dass bis zum Alter 29 kaum Unterschiede im Sterberisiko zwischen Ost- Westdeutschland existieren (Abbildung1). Ab dem Alter 30 bis 54 jedoch ist Ostdeutschland durch eine Übersterblichkeit gekennzeichnet, die allerdings nicht statistisch signifikant ist. Ab dem Alter 55 drehen sich die Verhältnisse schließlich um. Die Altersgruppen ab 55 Jahre sind in Westdeutschland durch ein vergleichsweise höheres Sterberisiko gekennzeichnet, hier machen sich eventuell die Selektionseffekte durch Ausschluss bestimmter Risiken bemerkbar. Dass in der gesamten Betrachtung der Sterblichkeit bei Kontrolle für Beschäftigung, Versicherung und Staatsangehörigkeit die Sterblichkeitsunterschiede zwischen Ost- und Westdeutschland fast vollständig verschwinden, ist somit Konsequenz dessen, dass sich Phasen der Über- und Untersterblichkeit über das Alter hinweg abwechseln. 


\section{Abbildung 1: Interaktionseffekt - Veränderung des Alterseffekts der Sterblich- keit in den Kategorien der Region; „Aktiv Versicherte“ Männer 2004}

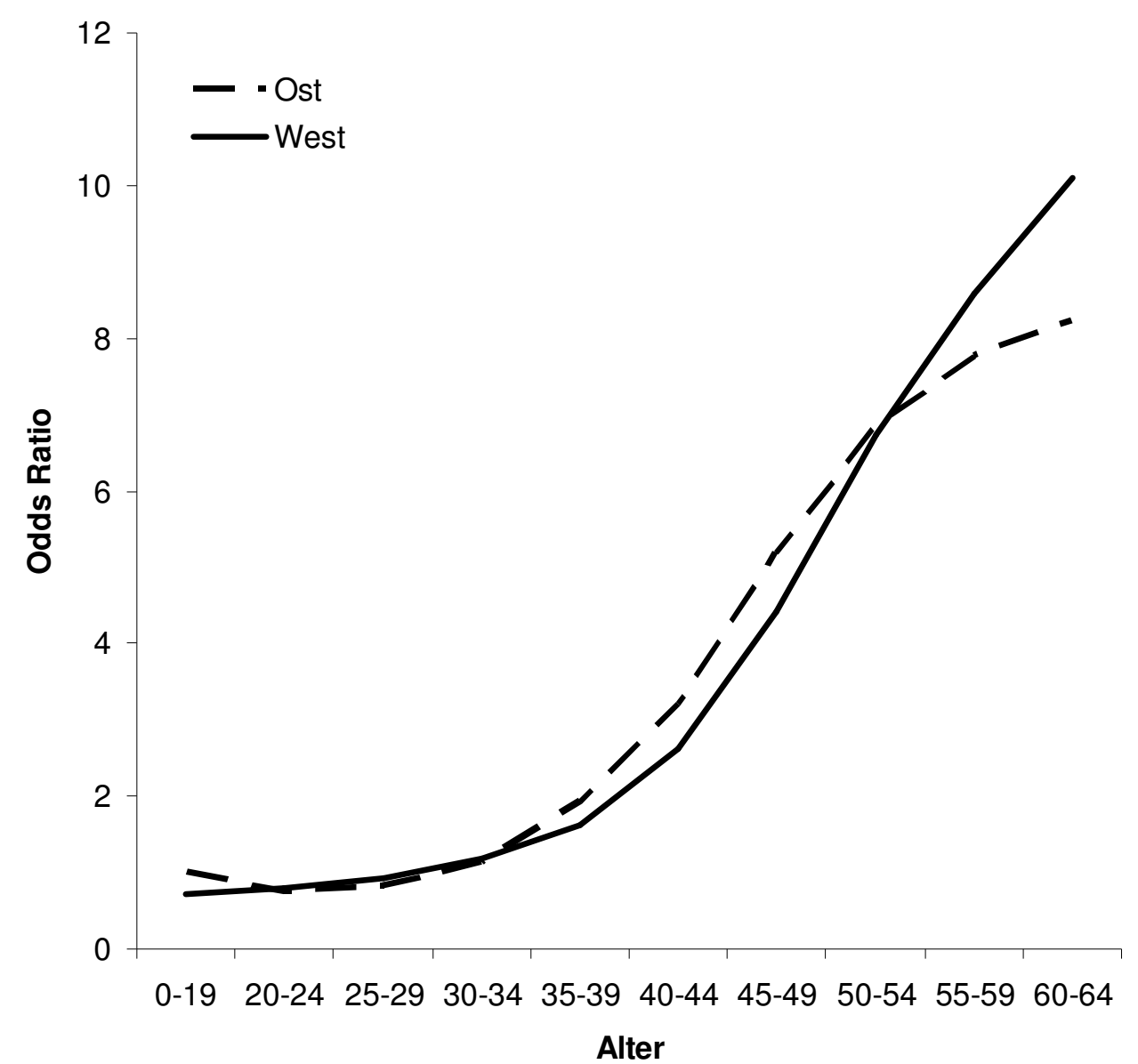

Quelle: FDZ-RV - Aktiv Versicherte 2004; „Ausgang_AKVS2004n.dat“ und „Abgang_AKVS2004n.dat“, Sonderauswertung, eigene Berechnungen.

Als Fazit der multivariaten Analyse ergibt sich: Die Untersuchung mit dem logistischen Regressionsmodell weist nach, dass die Unterschiede im Sterblichkeitsniveau zwischen Ost- und Westdeutschland weitestgehend durch Kompositionseffekte getragen werden. Die ostdeutsche Bevölkerung zeichnet sich hinsichtlich wichtiger Strukturmerkmale durch eine relativ ungünstigere Zusammensetzung aus, was insgesamt zu einer vergleichsweise höheren Sterblichkeit führt, wenn für diese Variablen nicht kontrolliert wird. Bei Kontrolle für die Merkmale Alter, Beschäftigung, Versicherung und Staatsangehörigkeit gleichen sich, wie Modell 4 zeigt, Ost- und Westdeutschland in ihrer Sterblichkeit nahezu an. Die Analyse des Interaktionseffektes zeigt, dass es in den mittleren Altersstufen eine geringe Übersterblichkeit von Ostdeutschland gegenüber Westdeutschland gibt, die allerdings keine statistische Signifikanz besitzt. Somit lässt sich festhalten, dass die ostdeutsche Übersterblichkeit mit den Daten der „Aktiv 
Versicherten“ vom FDZ-RV nahezu vollkommen durch Kompositionseffekte erklärt werden kann.

\section{Zusammenfassung}

Ziel des vorliegenden Beitrages ist es, die Differenzen der Lebenserwartung zwischen Ost- und Westdeutschland für Männer im Alter von 20 bis 65 Jahren nach Ursachen zu beleuchten. Für die Berechnung der Sterblichkeit wurden die Daten der „Aktiv Versicherten“ vom Forschungsdatenzentrum der Rentenversicherung (FDZ-RV) herangezogen, die fast $80 \%$ der männlichen Bevölkerung repräsentieren. Die deskriptive Analyse der Sterbewahrscheinlichkeiten zeigt, dass die ostdeutsche Übersterblichkeit nicht in allen Altersgruppen auftritt und nicht für alle Untergruppen des Beschäftigungsstatus und der Art der Tätigkeit gleichermaßen gilt. Besonders die mittleren Altersgruppen 35 bis 55 Jahre sind durch höhere Sterbewahrscheinlichkeiten in Ostdeutschland im Vergleich zu Westdeutschland gekennzeichnet. Für den Beschäftigungsstatus zeigt sich, dass vor allem die Gruppen, die Zeiten der Arbeitslosigkeit oder Selbstständigkeit aufweisen, sowie die Personen, für die keine Angabe zu diesem Merkmal vorliegt, die Übersterblichkeit in Ostdeutschland bedingen. Die Differenzierung nach dem Versicherungszweig stellt heraus, dass die ostdeutsche Übersterblichkeit vor allem von den Angehörigen der Arbeiterrentenversicherung und der Knappschaft getragen wird.

Die multivariate Analyse hat gezeigt, dass die ostdeutsche Übersterblichkeit vornehmlich Konsequenz einer im Vergleich zu Westdeutschland ungünstigeren Zusammensetzung der ostdeutschen Bevölkerung hinsichtlich Altersstruktur, Ausländeranteil und sozioökonomischen Faktoren (hier mit Beschäftigungsstatus, Arbeitslosigkeit und Art der Tätigkeit erfasst) ist. Wird für diese Merkmale kontrolliert, kann nahezu die gesamte Differenz in der Mortalität der Männer zwischen den beiden Regionen erklärt werden. Offensichtlich hat die allgemeine unterschiedliche Arbeitsmarktlage in Ost- und Westdeutschland den größten Einfluss auf die Differenz der Sterblichkeit. Bei gleicher Komposition der beiden Teilbevölkerungen gäbe es demnach keinen Sterblichkeitsunterschied. Bei einer Angleichung der Arbeitsmarktsituation in Ost- und Westdeutschland ist ein Rückgang der Differenzen der Mortalität bei Männern zu erwarten.

Die Ergebnisse decken sich mit anderen Untersuchungen in der Literatur, die bisher nur auf der Ebene von ökologischen Untersuchungen (z. B. Grözinger 2009) erzielt wurden. Jetzt kann der Nachweis der Wirkung von Arbeitslosigkeit auf Lebenserwartung auch auf der Individualebene für die gesamte Population nachgewiesen werden. 
Es zeigt sich, dass die Daten des FDZ-RV einen wichtigen Beitrag leisten können, empirische Befunde der Sterblichkeit in Deutschland aufzuhellen, was die Bedeutung des Zuganges zu solchen Routinestatistiken für die Forschung unterstreicht.

\section{Literatur}

Datensatz zur Statistik der aktiv Versicherten nach § 1 ABS.1 RSVwV ab der Erhebung zum 31.12.2004 Stand 8.März 2005 FDZ -RV Deutsche Rentenversicherung Bund (Editor)

Grözinger, G. (2009):

Achtung Lebensgefahr! Indirekte Effekte regionaler Arbeitslosigkeit auf Lebensweise und -Qualität. Intervention European Journal of Economics and Economic Policies $6(1), 2009,12-24$

Himmelreicher, R. K.; Sewöster, D.; Scholz, R.D. \& Schulz, A. (2008):

Die fernere Lebenserwartung von Rentnern und Pensionären im Vergleich. WSI Mitteilungen, 5/2008: 274-280.

Hosmer, D. W. \& Lemeshow, S. (1989):

Applied Logistic Regression. New York: Wiley- Intescience.

Mortalitäts-Forschungs-Datenbank: Human Mortality Database, http://www.mortality.org

Kibele, E. (2008):

Determinanten von regionalen Mortalitätsunterschieden in der Rentnerbevölkerung, Deutsche Rentenversicherung Bund (Editor): Etablierung und Weiterentwicklung: Bericht vom vierten Workshop des Forschungsdatenzentrums der Rentenversicherung (FDZ-RV) am 28. und 29. Juni $2007 \mathrm{im}$ Wissenschaftszentrum Berlin für Sozialforschung (WZB). Bad Homburg: WDV, 143-156.

Kibele, E. \& Scholz, R.D.(2009a):

Trend der Mortalitätsdifferenzen zwischen Ost und West unter Berücksichtigung der vermeidbaren Sterblichkeit; In: Cassens, I.; Luy, M. \& Scholz, R.: Die Bevölkerung in Ost- und Westdeutschland: Demografische, gesellschaftliche und wirtschaftliche Entwicklungen seit der Wende. Wiesbaden: VS Verlag für Sozialwissenschaften: 124139.

Kibele, E. \& Scholz, R.D. (2009b):

Verbesserte medizinische Versorgung zahlt sich aus: Lebenserwartungen in Ost- und Westdeutschland haben sich schnell angenähert, Demografische Forschung Aus Erster Hand, 6:3, 4-4. http://www.demografische-forschung.org/archiv/defo0903.pdf

Klein, T. (1993):

Soziale Determinanten der Lebenserwartung. Kölner Zeitschrift für Soziologie und Sozialpsychologie 45: 712-730.

Klein, T. \& Unger, R. (2001): Einkommen, Gesundheit und Mortalität in Deutschland, Großbritannien und den USA. Kölner Zeitschrift für Soziologie und Sozialpsychologie, 53: $96-110$. 
Luy, M. (2006):

Differentielle Sterblichkeit: die ungleiche Verteilung der Lebenserwartung in Deutschland. Rostocker Zentrum - Diskussionspapier 6.

http://www.rostockerzentrum.de/publikationen/rz diskussionpapier 6.pdf

Scholz, R.D. \& Schulz, A. (2009):

Haben Arbeitslosigkeit und Arbeitsunfähigkeit einen Einfluss auf die Höhe der Lebenserwartung?, Sozialwissenschaftlicher Fachinformationsdienst : Bevölkerungsforschung, Bd. 1/2009, S. 9-22; (http://

www.gesis.org/dienstleistungen/fachinformationen/servicepublikationen/sofid/themenbereiche/).

Scholz, R.D. \& Schulz, A. (2008):

Zum Trend der differentiellen Sterblichkeit der Rentner in Deutschland Deutsche Rentenversicherung Bund (Editor): Fünf Jahre FDZRV Bericht vom fünften Workshop des Forschungsdatenzentrums der Rentenversicherung (FDZ-RV)am 17. und 18. Juli 2008 im Wissenschaftszentrum Berlin für Sozialforschung (WZB),DRV-Schriften Bd. 55/2008, 144-152. http://forschung.deutscherentenversicherung.

de/ForschPortalWeb/ressource?key=DRV_Band_55_2009_Scholz_Schulz

Shkolnikov, V.M.; Scholz, R.D.; Jdanov, D.A.; Stegmann, M. non Gaudecker, H.M. (2008):

Length of life and the pensions of five million retired German men.

The European Journal of Public Health, 18(3). 264-269.

https://intranet.demogr.mpg.de/publications/files/2843 12030919281 Length\%20of\%20life.pdf

Wiesner, G. \& Bittner, E. K. (2004):

Lebenserwartung, vorzeitig verlorene Lebensjahre und vermeidbare Sterblichkeit im Ost-West-Vergleich. Bundesgesundheitsblatt- Gesundheitsforschung-Gesundheitsschutz 47: 266-278. 